PHYSICAL REVIEW D 92, 124051 (2015)

\title{
Nonlocal gravity in D dimensions: Propagators, entropy, and a bouncing cosmology
}

\author{
Aindriú Conroy, ${ }^{1}$ Anupam Mazumdar, ${ }^{1,2}$ Spyridon Talaganis, ${ }^{1}$ and Ali Teimouri ${ }^{1}$ \\ ${ }^{1}$ Consortium for Fundamental Physics, Lancaster University, Lancaster LA1 4YB, United Kingdom \\ ${ }^{2}$ IPPP, Durham University, Durham DH1 3LE, United Kingdom \\ (Received 16 September 2015; published 22 December 2015)
}

\begin{abstract}
We present the graviton propagator for an infinite derivative, $D$-dimensional, nonlocal action, up to quadratic order in curvature around a Minkowski background, and discuss the conditions required for this class of gravity theory to be ghost-free. We then study the gravitational entropy for de-Sitter and anti-de Sitter backgrounds, before comparing with a recently derived result for a Schwarzschild black hole, generalized to arbitrary $D$ dimensions, whereby the entropy is given simply by the area law. A novel approach of decomposing the entropy into its $(r, t)$ and spherical components is adopted in order to illustrate the differences more clearly. We conclude with a discussion of de-Sitter entropy in the framework of a nonsingular bouncing cosmology.
\end{abstract}

DOI: 10.1103/PhysRevD.92.124051

PACS numbers: 04.50.Kd, 04.50.-h, 04.60.Cf, 04.70.Dy

\section{INTRODUCTION}

Einstein's general relativity (GR) is a well-tested theory of gravity in the infrared (IR) [1], reducing to Newtonian gravity for nonrelativistic systems. At very short distances, the Newtonian potential has been tested only up to the distance scale of $10^{-5} \mathrm{~m}$, which is the IR limit of Einstein's gravity for a slowly time varying test mass [2]. The corresponding mass scale is around $M \sim 1 \mathrm{eV}$, which means that beyond this mass scale, the nature of gravity itself is very poorly constrained. As it stands, Einstein's theory is plagued by spacetime singularities - be it, the singularity of a black hole or the initial, cosmological singularity [3]. The latter is believed to be a naked singularity, which prevents, not only geodesic completeness for any null or time like trajectories, but also prevents us from comprehending the initial classical and quantum state of the Universe.

Einstein's gravity, however, is unique in a sense that it predicts the area-law of gravitational entropy for a gravitationally bound system. In 4-dimensions, the BekensteinHawking entropy of a blackhole is given by the area of a 2-dimensional horizon [4,5], which may also be confirmed by following Wald's prescription for the gravitational entropy of a static, homogeneous and isotropic metric, supplemented with a horizon [6], see also [7]. This observation has lead to many well-known conjectures, such as the famous anti-deSitter (AdS) conformal field theory (CFT) correspondence [8], and the holographic principle, which states that the gravitational entropy is proportional to the surface area rather than the volume $[9,10]$. The illusory nature of the volume and the subsequent holographic quality of the Universe, itself, affords the principle its name.

Of course, some of the concerns raised by Einstein's gravity at short distances and small time scales need to be addressed by better understanding the ultraviolet (UV) aspects of gravity, while retaining the aforementioned predictions in the IR. However, the challenges in modifying gravity arises from two fronts. On one hand, higher-order curvature corrections tend to ameliorate the UV aspects by suppressing the graviton propagator. On the other, gravity being a derivative theory, higher-order corrections naturally introduce extra propagating states, which can be massive, tachyonic or ghost-like. A classic example is Stelle's 4th derivative gravity which is renormalizable, but contains a massive spin-2 ghost [11].

In Refs. [12,13], the issue of ghosts has been addressed at tree-level in 4-dimensions. An action can be rendered ghost-free by requiring that no additional degrees of freedom other than the massless graviton are introduced. ${ }^{1}$ This is in spite of the fact that there are an infinite number of derivatives acting on the curvature. The action, in fact, remains generally covariant, giving rise to nonlocal graviton interactions, and has improved UV behavior in terms of higher loops [14], see also $[15,16]$.

A further observation was made by studying the true dynamical degrees of freedom propagating between the spacetime region and the gravitational entropy of a selfgravitating system, containing a horizon. In Ref. [17], it was shown that the gravitational entropy of such a nontrivial, ghost-free gravitational action for a Schwarzschild's black hole in 4 dimensions is determined solely by the Einstein-Hilbert action. The higher-order corrections, at least up to quadratic in curvature, do not contribute to the gravitational entropy.

The aim of this paper is two-fold.

First, we wish to:

\footnotetext{
${ }^{1}$ More generally, one simply requires that the modified propagator is proportional to the propagator of GR. In such a case, it is possible, within certain limitations, to introduce an additional scalar propagating degree of a freedom and for the theory to remain ghost-free.
} 
(i) Extend the results of Refs. [12] to $D$-spacetime dimensions, by detailing the graviton propagator and the subsequent constraints for a ghost-free construction of gravity in $D$ dimensions around Minkowski background.

(ii) Generalise the entropy computation for a Schwarzschild-like black hole to $D$ dimensions and show that the entropy for such a setup will be determined solely by the Einstein-Hilbert action [17].

And second, we intend to

(i) Study the gravitational entropy for a de-Sitter (dS) and anti-de-Sitter (AdS) metric, again in $D$-spacetimedimensions.

(ii) Discuss this result in the context of a nonsingular bouncing cosmology.

Furthermore, note that in this paper we do not attempt to present the graviton propagator for (A)dS, leaving this for future study.

The paper is organized as follows: In Sec. II, we provide some technical details on how to obtain the graviton propagator in $D$-spacetime dimensions and study the ghost-free condition around Minkowski background. In Sec. III, we discuss the general definition of gravitational entropy by following Wald's prescription. In Sec. IV, we present the gravitational entropy for a $D$ dimensional Schwarzschild-like blackhole. In Sec. V, we study the gravitational entropy for dS and AdS in $D$ dimensions, and in Sec. VI, we apply this result in a cosmological context.

\section{INFINITE DERIVATIVE GRAVITY IN $D$ DIMENSIONS}

The most general, ${ }^{2} D$-dimensional, nonlocal action of gravity that is quadratic in curvature can be expressed as a combination of the Einstein-Hilbert term and higher order terms ${ }^{3}$

$$
\begin{aligned}
I^{\mathrm{tot}}= & \frac{1}{16 \pi G_{D}} \int d^{D} x \sqrt{-g}\left[R+\alpha\left(R \mathcal{F}_{1}(\square) R\right.\right. \\
& \left.\left.+R_{\mu \nu} \mathcal{F}_{2}(\square) R^{\mu \nu}+R_{\mu \nu \lambda \sigma} \mathcal{F}_{3}(\square) R^{\mu \nu \lambda \sigma}\right)\right],
\end{aligned}
$$

where $G_{D}$ is the $D$-dimensional Newton's constant ${ }^{4} ; \alpha$ is a constant $^{5}$ with dimension of inverse mass squared; and $\mu, \nu, \lambda, \sigma$ run from $0,1,2, \cdots D-1$. We briefly note that actions of higher order in curvature are permitted by the

\footnotetext{
${ }^{2}$ We are considering theories of gravity that are parity invariant and torsion-free.

${ }^{3}$ The structure is very similar to Ref. [12], which was derived in 4 dimensions.

${ }^{4}$ In $D$ dimension $G_{D}$ has dimension of $\left[G^{(D)}\right]=\left[G^{(4)}\right] L^{D-4}$ where $\mathrm{L}$ is unit length.

${ }^{5}$ Note that for an arbitrary choice of $\mathcal{F}(\square)$ at action level, $\alpha$ can be positive or negative as one can absorbs the sign into the coefficients $f_{i_{n}}$ contained within $\mathcal{F}(\square)$ to keep the overall action unchanged, however $\alpha$ has to be strictly positive once we impose ghost-free condition [18].
}

general diffeomorphism, however, we restrict ourself to actions of quadratic order, which capture all the quadratic perturbations, around the Minkowski spacetime, required for studying the graviton propagator $[12,13]$. The form factors given by $\mathcal{F}_{i}(\square)$ contain an infinite number of covariant derivatives, of the form:

$$
\mathcal{F}_{i}(\square) \equiv \sum_{n=0}^{\infty} f_{i_{n}}\left(\frac{\square}{M^{2}}\right)^{n}
$$

with constants $f_{i_{n}}$, and $\square \equiv g^{\mu \nu} \nabla_{\mu} \nabla_{\nu}$ is the D'Alembertian operator. The reader should note that, in our presentation, the function $\mathcal{F}_{i}(\square)$ comes with an associated $D$-dimensional mass scale, $M \leq M_{p}=\left(1 / \sqrt{\left(8 \pi G_{D}\right)}\right)$, which determines the scale of nonlocality in a quantum sense, see [14]. The full classical equations of motion has been derived for action Eq. (1) in 4 dimensions in Ref. [19], see also [20] for mathematical techniques.

In particular, let us consider perturbations around $D$-dimensional Minkowski spacetime with metric tensor $\eta_{\mu \nu}$, such that $\eta_{\mu \nu} \eta^{\mu \nu}=D$, and where the perturbations are denoted by $h_{\mu \nu}$. One should also note that we are using mostly plus metric signature convention.

The $\mathcal{O}(h)$ expressions for the Riemann tensor, Ricci tensor and curvature scalar in $D$ dimensions are given by:

$$
\begin{aligned}
R_{\mu \nu \lambda \sigma} & =\frac{1}{2}\left(\partial_{[\lambda} \partial_{\nu} h_{\mu \sigma]}-\partial_{[\lambda} \partial_{\mu} h_{\nu \sigma]}\right) \\
R_{\mu \nu} & =\frac{1}{2}\left(\partial_{\sigma} \partial_{(\nu} \partial_{\mu)}^{\sigma}-\partial_{\mu} \partial_{\nu} h-\square h_{\mu \nu}\right) \\
R & =\partial_{\mu} \partial_{\nu} h^{\mu \nu}-\square h .
\end{aligned}
$$

The full action Eq. (1) can then be expanded around Minkowski space, retaining terms up to $\mathcal{O}\left(h^{2}\right)$ :

$$
\begin{aligned}
S_{q}= & -\int d^{D} x\left[\frac{1}{2} h_{\mu \nu} \square a(\square) h^{\mu \nu}+h_{\mu}^{\sigma} b(\square) \partial_{\sigma} \partial_{\nu} h^{\mu \nu}\right. \\
& +h c(\square) \partial_{\mu} \partial_{\nu} h^{\mu \nu}+\frac{1}{2} h \square d(\square) h \\
& \left.+h^{\lambda \sigma} \frac{f(\square)}{2 \square} \partial_{\sigma} \partial_{\lambda} \partial_{\mu} \partial_{\nu} h^{\mu \nu}\right] .
\end{aligned}
$$

The above expression, along with the functional forms of $a(\square), b(\square), c(\square), d(\square)$ and $f(\square)$ remain the same as those of the 4-dimensional case, see Ref. [12,13], and our Appendix A. One can easily note that

$$
f(\square)=a(\square)-c(\square),
$$

and that the equation of motion satisfies the generalized Bianchi identities, for energy momentum tensor $\tau_{\mu \nu}$ : 


$$
\begin{aligned}
\nabla_{\mu} \tau_{\nu}^{\mu}= & 0=(c+d) \square \partial_{\nu} h \\
& +(a+b) \square h_{\nu, \mu}^{\mu}+(b+c+f) h_{, \alpha \beta \nu}^{\alpha \beta} .
\end{aligned}
$$

To find the graviton propagator in $D$ dimensions, we follow a similar projection operator prescription as found in Refs. [12,13,21], see also Appendix B.

Thus the $D$-dimensional propagator is now given by

$$
\Pi\left(-k^{2}\right)=\frac{\mathcal{P}^{2}}{k^{2} a\left(-k^{2}\right)}+\frac{\mathcal{P}_{s}^{0}}{k^{2}\left(a\left(-k^{2}\right)-(D-1) c\left(-k^{2}\right)\right)}
$$

Choosing $f(\square)=0 \Rightarrow a(\square)=c(\square)$, so as not to introduce any scalar propagating degrees of freedom, we find

$$
\Pi=\frac{1}{k^{2} a\left(-k^{2}\right)}\left(\mathcal{P}^{2}-\frac{1}{D-2} \mathcal{P}_{s}^{0}\right) .
$$

The form of $a\left(-k^{2}\right)$ should be such that it does not introduce any new propagating degree of freedom, and it was argued in Refs. [12,18] that the form of $a(\square)$ should be an entire function, so as not to introduce any pole in the complex plane, which would result in additional degrees of freedom in the momentum space ${ }^{6}$.

Furthermore, the form of $a\left(-k^{2}\right)$ should be such that in the IR, for $k \rightarrow 0, a\left(-k^{2}\right) \rightarrow 1$, therefore recovering the propagator of GR in the $D$ dimensions. For $D=4$, the propagator has the familiar $1 / 2$ factor in front of the scalar part of the propagator. One such example of an entire function is $[12,18]$ :

$$
a(\square)=e^{-\square / M^{2}},
$$

which has been found to ameliorate the UV aspects of gravity by removing the black hole singularity within the linearized limit in a static configuration [12], see also $[22,23]$, while recovering the Newtonian limit in the IR. ${ }^{7}$ In Refs. $[24,25]$, the authors have shown that the spherical collapse within a linearized limit does not form a curvature singularity in this class of models.

By imposing $f(\square)=0$, i.e. $a(\square)=c(\square)$, we obtain:

$$
2 \mathcal{F}_{1}(\square)+\mathcal{F}_{2}(\square)+2 \mathcal{F}_{3}(\square)=0
$$

which is independent of the number of spacetime dimensions. This result was first shown in Ref. [12] in 4 dimensions.

\footnotetext{
${ }^{6}$ Similar arguments for a propagator were also made in Refs. [15] before ours.

${ }^{7}$ In principle one can take $a(\square)=e^{\left(-\square / M^{2}\right)^{n}}$, where $n$ is an integer, but a wrong choice of sign for instance $a(\square)=e^{-\left(-\square / M^{2}\right)^{n}}$ would not yield the correct Newtonian potential [14].
}

\section{GRAVITATIONAL ENTROPY}

In the framework of Lagrangian field theory, Wald showed that one can find the gravitational entropy by varying the Lagrangian and subsequently finding the Noether current as a function of an assigned vector field. By writing the corresponding Noether charge, it has been shown that, for a static blackhole, the first law of thermodynamics can be satisfied and the entropy may be expressed by integrating the Noether charge over a bifurcation surface of the horizon. In so doing, one must choose the assigned vector field to be a horizon Killing vector, which has been normalized to unit surface gravity, see [6,7].

In order to compute the gravitational entropy of the nonlocal theory outlined above, we impose a $D$-dimensional, static, homogenous and spherically symmetric metric, containing a horizon, of the form

$$
d s^{2}=-f(r) d t^{2}+f(r)^{-1} d r^{2}+r^{2} d \Omega_{D-2}^{2} .
$$

This metric is equally applicable to (A)dS metric as it is to the static black hole case, and results in the following entropy equation,

$$
S_{W}=-8 \pi \oint_{r=r_{H}, t=\mathrm{const}} \frac{\delta \mathcal{L}}{\delta R_{\mathrm{rttr}}} r^{D-2} d \Omega_{D-2}^{2},
$$

where the bifurcation surface is at $\left(r=r_{H}\right), t=$ const. The area of the horizon, $\left(A_{H}\right)$, is given by integrating over the $(D-2)$-sphere as,

$$
A_{H}=\oint_{r=r_{H}, t=\mathrm{const}} r^{D-2} d \Omega_{D-2}^{2} .
$$

In the following sections, we first derive the $D$-dimensional entropy, corresponding to action Eq. (1), before turning our attention to the case of a static black hole and, subsequently, the dS and AdS cases.

By varying the Lagrangian density with respect to the Riemann tensor in $(r, t)$ directions, as given in Eq. (12), one may compute the entropy of the gravitational system described by the action Eq. (1) and corresponding metric Eq. (11), as follows

$$
\begin{aligned}
S_{W}= & \frac{A_{H}}{4 G_{D}}\left[1+\alpha\left(2 \mathcal{F}_{1}(\square) R-\mathcal{F}_{2}(\square) \times\left(g^{r r} R^{t t}+g^{r r} R^{r r}\right)\right.\right. \\
& \left.\left.-4 \mathcal{F}_{3}(\square) R^{\mathrm{rtrt}}\right)\right] .
\end{aligned}
$$

It is convenient, for illustrative purposes, to decompose the entropy equation into its $(r, t)$ and spherical components. For a static and axis-symmetric metric Eq. (11), we denote the $(r)$ and $(t)$ directions by the indices $\{a, b\}$; and the spherical components by $\{\bar{m}, \bar{n}\}$. As such, we express the curvature scalar as follows 


$$
R=g^{\mu \nu} R_{\mu \nu}=g^{a b} R_{a b}+g^{\bar{m} \bar{n}} R_{\bar{m} \bar{n}},
$$

where $g_{a b}$ is a 2-dimensional metric tensor accounting for the $r, t$ directions and $g_{\bar{m} \bar{n}}$ is a $(D-2)$-dimensional metric tensor, corresponding to the angular components, such that

$$
g^{\mu \nu} g_{\mu \nu} \equiv g^{a b} g_{a b}+g^{\bar{m} \bar{n}} g_{\bar{m} \bar{n}}=2+(D-2)=D .
$$

Expanding the scalar curvature into Ricci and Riemann tensors, along with the properties of the static, spherically symmetric metric Eq. (11), allows us to express the relevant components of the entropy equation as follows

$$
\begin{aligned}
g^{r r} R^{t t}+g^{r r} R^{r r} & =-g_{t t} R^{t t}-g_{r r} R^{r r}=-g^{a b} R_{a b} \\
-4 R_{r t r t} & =2 g^{a b} R_{a b}-2 g^{a b} g^{\bar{m} \bar{n}} R_{\bar{m} a \bar{n} b} .
\end{aligned}
$$

Substitution into Eq. (14), results in a decomposed $D$-dimensional entropy equation for the action (1) in a static, spherically symmetric background

$$
\begin{aligned}
S_{W}= & \frac{A_{H}}{4 G_{D}}\left[1+\alpha\left(2 \mathcal{F}_{1}(\square)+\mathcal{F}_{2}(\square)+2 \mathcal{F}_{3}(\square)\right) g^{a b} R_{a b}\right. \\
& \left.+2 \alpha\left(\mathcal{F}_{1}(\square) g^{\bar{m} \bar{n}} R_{\bar{m} \bar{n}}-\mathcal{F}_{3}(\square) g^{a b} g^{\bar{m} \bar{n}} R_{\bar{m} a \bar{n} b}\right)\right] .
\end{aligned}
$$

This decomposed form is particular illustrative in the context of a static axis-symmetric blackhole.

\section{D-DIMENSIONAL BLACK HOLE ENTROPY}

For a spherically symmetric $D$-dimensional background, the angular components of the Ricci tensor are given by $R_{\theta_{n} \theta_{n}}=\sin ^{-2}\left(\theta_{n}\right) R_{\theta_{n+1} \theta_{n+1}}$, where $\theta_{n}$ runs from 1 to $D-2$, signifying each angular direction. Explicitly, for the given metric, $R_{\theta_{1} \theta_{1}}$ is given by

$$
R_{\theta_{1} \theta_{1}}=(D-3)-(D-3) f(r)-r f^{\prime}(r)=0,
$$

for a vacuum solution, and solving the straightforward differential equation, one reveals

$$
f(r)=1-\frac{\mu}{r^{D-3}},
$$

where $\mu$ is a constant of integration. The form of this function and its associated metric, encompasses the $D$ dimensional analogue of the Schwarzschild solution, known as the Schwarzschild-Tangherlini metric [26], with $\mu=\frac{16 \pi G_{D} M}{(D-2) A_{D-2}}$, [27]; and is an asymptotically $D$-dimensional Minkowski background.

Thus, when considering a Schwarzschild solution, all $R_{\theta_{i} \theta_{i}}$ components, will vanish. This is a consequence of the axisymmetric properties of the solution. Therefore, the entropy of a $D$-dimensional static and spherically symmetric metric with a horizon, yields:
$S_{W}=\frac{A_{H}}{4 G_{D}}\left[1+\alpha\left(2 \mathcal{F}_{1}(\square)+\mathcal{F}_{2}(\square)+2 \mathcal{F}_{3}(\square)\right) g^{a b} R_{a b}\right]$.

Combining the above with the constraint given in Eq. (10), results in the gravitational entropy of the modified sector vanishing entirely. Thus, the $D$-dimensional blackhole entropy corresponding to action Eq. (1) is given solely by the Bekenstein-Hawking area law

$$
S_{W}=\frac{A_{H}}{4 G_{D}} .
$$

This simple observation ensures that, in the context of a static, spherically symmetric metric, which asymptotes to Minkowski, the holographic nature of gravity is preserved in the IR. The higher-order corrections to the UV do not affect the gravitational entropy stored on the horizon, as long as the only propagating degrees of freedom are the massless graviton. To some extent, one may be able to conjecture that the area-law of gravitational entropy is purely an IR concept of nature in such circumstances.

This is a powerful result. For instance, any arbitrary $f(R)$ theory of gravity would contribute toward the gravitational entropy for the above choice of metric. We can understand this very simply because such a class of theory would induce an extra scalar propagating degree of freedom other than the massless graviton, which would contribute toward the gravitational entropy.

In some sense, there is an intriguing connection between propagating degrees of freedom and the gravitational entropy at the horizon, at least in the context of a static, spherically symmetric background, which asymptotes to Minkowski. This elegant result may not hold for a (A)dS background as we shall see below, since the form of the propagator for infinite derivative theory of gravity will be different for dS and AdS compared to Minkowski.

\section{D-DIMENSIONAL (A)dS ENTROPY}

We now turn our attention to another class of metrics which contain a horizon, such as the (A)dS metrics, where the $D$-dimensional nonlocal action Eq. (1) must now be appended with a cosmological constant $\Lambda$ to ensure that (A)dS is a vacuum solution.

$$
\begin{aligned}
I^{\mathrm{tot}}= & \frac{1}{16 \pi G_{D}} \int d^{D} x \sqrt{-g}\left[R-2 \Lambda+\alpha\left(R \mathcal{F}_{1}(\square) R\right.\right. \\
& \left.\left.+R_{\mu \nu} \mathcal{F}_{2}(\square) R^{\mu \nu}+R_{\mu \nu \lambda \sigma} \mathcal{F}_{3}(\square) R^{\mu \nu \lambda \sigma}\right)\right] .
\end{aligned}
$$

The cosmological constant is then given by

$$
\Lambda= \pm \frac{(D-1)(D-2)}{2 l^{2}},
$$


where the positive sign corresponds to dS, negative to AdS, and hereafter, the topmost sign will refer to dS and the bottom to AdS. $l$ denotes the cosmological horizon. The (A)dS metric can be obtained by taking

$$
f(r)=\left(1 \mp \frac{r^{2}}{l^{2}}\right),
$$

in Eq. (11).

Recalling the $D$-dimensional entropy Eq. (18), we write,

$$
\begin{aligned}
S_{W}^{(\mathrm{A}) \mathrm{dS}}= & \frac{A_{H}^{(\mathrm{A}) \mathrm{dS}}}{4 G_{D}}\left[1+\alpha\left(2 \mathcal{F}_{1}(\square)+\mathcal{F}_{2}(\square)+2 \mathcal{F}_{3}(\square)\right)\right. \\
& \times g^{a b} R_{a b}+2 \alpha\left(\mathcal{F}_{1}(\square) g^{\bar{m} \bar{n}} R_{\bar{m} \bar{n}}\right. \\
& \left.\left.-\mathcal{F}_{3}(\square) g^{a b} g^{\bar{m} \bar{n}} R_{\bar{m} a \bar{n} b}\right)\right],
\end{aligned}
$$

where now $A_{H}^{(\mathrm{A}) \mathrm{dS}} \equiv l^{D-2} A_{D-2}$, with $A_{D-2}=\left(2 \pi^{\frac{D-1}{2}}\right) /$ $\Gamma\left[\frac{D-1}{2}\right]$. Given the $D$-dimensional definitions of curvature in (A)dS background,

$$
\begin{aligned}
R_{\mu \nu \lambda \sigma} & = \pm \frac{1}{l^{2}} g_{[\mu \lambda} g_{\nu] \sigma}, \quad R_{\mu \nu}= \pm \frac{D-1}{l^{2}} g_{\mu \nu}, \\
R & = \pm \frac{D(D-1)}{l^{2}},
\end{aligned}
$$

simple substitution reveals the gravitational entropy in (A)dS can be expressed as:

$$
\begin{aligned}
S_{W}^{(\mathrm{A}) \mathrm{dS}}= & \frac{A_{H}^{(\mathrm{A}) \mathrm{dS}}}{4 G_{D}}\left(1 \pm \frac{2 \alpha}{l^{2}}\left\{f_{1_{0}} D(D-1)\right.\right. \\
& \left.\left.+f_{2_{0}}(D-1)+2 f_{3_{0}}\right\}\right) .
\end{aligned}
$$

Note that $f_{i_{0}}$ 's are now simply the leading constants of the functions $\mathcal{F}_{i}(\square)$, due to the nature of curvature in (A) dS. In particular, in 4-dimensions, the combination $12 f_{1_{0}}+$ $3 f_{2_{0}}+2 f_{3_{0}}$ is very different from that of the Minkowski space constraint, see Eq. (10), required for the massless nature of a graviton in any $D$ dimensions around Minkowski. Deriving the precise form of the ghost-free constraint in (A)dS, is still an open problem for the above action Eq. (1).

\section{NONSINGULAR BOUNCING COSMOLOGY AND HOLOGRAPHIC ENTROPY}

One of the applications of seeking (A)dS gravitational entropy for an infinite derivative theory of gravity is to understand the initial conditions for the Universe. It has been known for some time that nonlocality in gravity resolves the cosmological singularity problem, at least in the context of homogeneous and isotropic metric, such as Friedmann-Robertson-Walker (FRW) background [18].
Even small inhomogeneities, i.e. sub- [28] and superHubble [29] perturbations around such a non-singular bouncing solution are stable. ${ }^{8}$

A subclass of the full action Eq. (23) in 4 dimensions is given by ${ }^{9}$ :

$I_{R}=\frac{1}{16 \pi G_{4}} \int d^{4} x \sqrt{-g}\left(R-2 \Lambda+\alpha R \mathcal{F}_{1}(\square) R\right)$,

where $\alpha>0$ is required to ensure that gravity remains ghost-free. A reduced action of this type has been studied in [32], where it was shown in a FRW spacetime (consequently dS), that null rays can be made past-complete without violating any relevant energy conditions [32], thus replacing the cosmological singularity with a bounce at $t=0$.

It was shown in [18] that a spacetime may be rendered ghost-free for the following choice of $\mathcal{F}(\square)$ :

$$
\mathcal{F}_{1}(\square)=\frac{e^{-\square / M^{2}}-1}{\square / M^{2}} .
$$

A well-defined background solution which would satisfy the equations of motion for the above action Eq. (29) is given by $[28,29]$

$$
a(t)=a_{0} \cosh \left(\sqrt{\frac{\Lambda}{3}} t\right),
$$

where typically $\Lambda^{1 / 2} \approx M$, i.e. the scale of nonlocality, $a_{0}$ is just a constant, and $H=l^{-1}=\sqrt{\Lambda / 3}$. At the bounce, $\dot{a}=0, \ddot{a}>0$, where dot denotes time derivative with respect to physical time $t$. The Universe loiters around a $\mathrm{dS}$ phase - the duration of which depends on how long the bouncing phase lasts around $t=0$.

It would indeed be worthwhile to ask what the gravitational entropy stored in a cosmological constant dominated Universe should be, at the time of the bounce. ${ }^{10}$ From Eq. (28), we read off the entropy equation for the action Eq. (29) in de Sitter space ${ }^{11}$

\footnotetext{
${ }^{8}$ In order to resolve the cosmological singularity in an asymptotically Minkowski background, one would need to depart from the condition $a(\square)=c(\square)$ in (B20). However, as alluded to in footnote 1 , the resulting propagator must be proportional to the GR propagator in order to be ghost-free. This is also reflected in a Friedmann-Robertson-Walker (FRW) background, where one would require an extra scalar degree of freedom besides the massless graviton [18].

${ }^{9}$ This action has also been proposed as a UV complete action for Starobinsky inflation [30,31].

${ }^{10}$ In the classical Einstein gravity this question is ill-definedwithout violating the energy conditions it is not possible to avoid the cosmological singularity.

${ }^{11}$ In AdS, the equivalent entropy of Eq. (32), is given by $S_{W}^{\mathrm{AdS}}=\frac{A_{H}^{\mathrm{AdS}}}{4 G_{4}}\left(1+\frac{24 \alpha}{l^{2}}\right)$.
} 


$$
S_{R}^{\mathrm{dS}}=\frac{A_{H}^{\mathrm{dS}}}{4 G_{4}}\left(1-\frac{24 \alpha}{l^{2}}\right) .
$$

\section{DISCUSSION AND CONCLUSION}

The first thing to note here is that modified gravitational entropy in a cosmological constant dominated background is diminished with respect to the entropy of Einstein's gravity. Furthermore, one notes that for a particular value of the arbitrary dimensionful quantity $\alpha=\frac{l^{2}}{24}$, the entropy vanishes entirely. This is in contrast to the black hole case where the UV modified sector does not contribute to the gravitational entropy, when no extra degrees of freedom are introduced into the system. In this case, the possibility that the gravitational entropy will vanish in its entirety, is allowed for. This is an intriguing outcome of infinite derivative nonlocal gravity.

It also raises the question: Could our Universe have begun its journey with a zero gravitational entropy? At the present moment, we merely speculate on the notion of a zero gravitational entropy state at the bounce point of cosmology. A zero entropy state for any system would be equivalent to realizing a ground state of the system. In our case, it is the graviton which realizes its ground state in the presence of $\Lambda$ and nonlocal gravity. Could this lead to a new state of gravity such that our Universe would yield a condensation of gravitons, at the moment of bounce, similar to the Bose-Einstein condensate with a zero entropy state [33]?

Some of these issues are indeed fundamental in nature and would perhaps open up new vistas toward understanding the nature of quantum aspects of gravity in extreme conditions and indeed, the nature of thermodynamics around the bounce.

In summary, we first demonstrated that in requiring that an infinite derivative theory of gravity, up to quadratic in curvature, is covariant and ghost-free, a constraint on the form factors $\mathcal{F}_{i}$ is revealed, given by Eq. (10). The relationship holds in any arbitrary $D$ dimensions. This is an extension of previous works, see [12], where the results were known to hold only in 4 dimensions. We further obtained the graviton propagator for such infinite derivative theory of gravity in $D$ dimensions such that the graviton remains massless and free from tachyon and ghosts.

We then studied the gravitational entropy at the horizon for an infinite derivative theory of gravity for a $D$-dimensional blackhole solution, i.e. a static and axissymmetric metric. We confirmed the area-law of gravitational entropy and reinstated the connection between the ghost-free condition for gravitons and the holographic nature of gravity in the IR.

We computed the gravitational entropy for a $D$ dimensions $\mathrm{A}(\mathrm{dS})$ metrics for such an infinite derivative theory of gravity. Unlike the black hole case, the gravitational entropy due to extra contributions from the UV does not vanish. As an application, we studied the gravitational entropy of a nonsingular bouncing cosmology at the bounce point, where we speculated upon the vanishing of gravitational entropy in its entirety.

\section{ACKNOWLEDGMENTS}

The authors would wish to thank Alex Koshelev and Tirthabir Biswas for numerous discussions. A. C. is funded by STFC Grant No. ST/K50208X/1, A. M. is supported by the STFC Grant No. ST/J000418/1 and S. T. is supported by a scholarship from the Onassis Foundation.

\section{APPENDIX A: BIANCHI IDENTITIES}

The $\mathcal{O}\left(h^{2}\right)$ part of the full action can be written as in Eq. (4)

$$
\begin{aligned}
S_{q}= & -\int d^{D} x\left[\frac{1}{2} h_{\mu \nu} \square a(\square) h^{\mu \nu}+h_{\mu}^{\sigma} b(\square) \partial_{\sigma} \partial_{\nu} h^{\mu \nu}\right. \\
& +h c(\square) \partial_{\mu} \partial_{\nu} h^{\mu \nu}+\frac{1}{2} h \square d(\square) h \\
& \left.+h^{\lambda \sigma} \frac{f(\square)}{2 \square} \partial_{\sigma} \partial_{\lambda} \partial_{\mu} \partial_{\nu} h^{\mu \nu}\right] .
\end{aligned}
$$

where we have,

$$
\begin{aligned}
R \mathcal{F}_{1}(\square) R= & \mathcal{F}_{1}(\square)\left[h \square \square^{2} h+h^{\lambda \sigma} \partial_{\sigma} \partial_{\lambda} \partial_{\mu} \partial_{\nu} h^{\mu \nu}\right. \\
- & \left.2 h \square \partial_{\mu} \partial_{\nu} h^{\mu \nu}\right] \\
R_{\mu \nu} \mathcal{F}_{2}(\square) R^{\mu \nu}= & \mathcal{F}_{2}(\square)\left[\frac{1}{4} h \square^{2} h+\frac{1}{4} h_{\mu \nu} \square^{2} h^{\mu \nu}\right. \\
& -\frac{1}{2} h_{\mu}^{\sigma} \square \partial_{\sigma} \partial_{\nu} h^{\mu \nu}-\frac{1}{2} h \square \partial_{\mu} \partial_{\nu} h^{\mu \nu} \\
+ & \left.\frac{1}{2} h^{\lambda \sigma} \partial_{\sigma} \partial_{\lambda} \partial_{\mu} \partial_{\nu} h^{\mu \nu}\right] \\
R_{\mu \nu \lambda \sigma} \mathcal{F}_{3}(\square) R^{\mu \nu \lambda \sigma}= & \mathcal{F}_{3}(\square)\left[h_{\mu \nu} \square{ }^{2} h^{\mu \nu}-2 h_{\mu}^{\sigma} \square \partial_{\sigma} \partial_{\nu} h^{\mu \nu}\right. \\
& \left.+h^{\lambda \sigma} \partial_{\sigma} \partial_{\lambda} \partial_{\mu} \partial_{\nu} h^{\mu \nu}\right]
\end{aligned}
$$

it should be noted that no $\eta_{\mu \nu} \eta^{\mu \nu}$ contractions appear. Hence, $a(\square), b(\square), c(\square), d(\square)$ and $f(\square)$ are as follows:

$$
\begin{aligned}
& a(\square)=1-\frac{1}{2} \mathcal{F}_{2}(\square) \frac{\square}{M^{2}}-2 \mathcal{F}_{3}(\square) \frac{\square}{M^{2}}, \\
& b(\square)=-1+\frac{1}{2} \mathcal{F}_{2}(\square) \frac{\square}{M^{2}}+2 \mathcal{F}_{3}(\square) \frac{\square}{M^{2}}, \\
& c(\square)=1+2 \mathcal{F}_{1}(\square) \frac{\square}{M^{2}}+\frac{1}{2} \mathcal{F}_{2}(\square) \frac{\square}{M^{2}}, \\
& d(\square)=-1-2 \mathcal{F}_{1}(\square) \frac{\square}{M^{2}}-\frac{1}{2} \mathcal{F}_{2}(\square) \frac{\square}{M^{2}},
\end{aligned}
$$


$f(\square)=-2 \mathcal{F}_{1}(\square) \frac{\square}{M^{2}}-\mathcal{F}_{2}(\square) \frac{\square}{M^{2}}-2 \mathcal{F}_{3}(\square) \frac{\square}{M^{2}}$.

We observe that

$$
\begin{gathered}
a(\square)+b(\square)=0, \\
c(\square)+d(\square)=0, \\
b(\square)+c(\square)+f(\square)=0 .
\end{gathered}
$$

Assuming $f(\square)=0$, we get that $a(\square)=c(\square)$ and hence the earlier constraint, $2 \mathcal{F}_{1}(\square)+\mathcal{F}_{2}(\square)+2 \mathcal{F}_{3}(\square)=0$. The generalized Bianchi identities give

$$
\begin{aligned}
\nabla_{\mu} \tau_{\nu}^{\mu}= & 0=(c+d) \square \partial_{\nu} h \\
& +(a+b) \square h_{\nu, \mu}^{\mu}+(b+c+f) h_{, \alpha \beta \nu}^{\alpha \beta},
\end{aligned}
$$

from which we can verify the constraints (A10)-(A12).

\section{APPENDIX B: SPIN PROJECTION OPERATORS IN $D$ DIMENSIONAL MINKOWSKI SPACE}

Now the spin projector operators in $D$ dimension Minkowski space are, see [13,21]

$$
\begin{gathered}
\mathcal{P}^{2}=\frac{1}{2}\left(\theta_{\mu \rho} \theta_{\nu \sigma}+\theta_{\mu \sigma} \theta_{\nu \rho}\right)-\frac{1}{D-1} \theta_{\mu \nu} \theta_{\rho \sigma}, \\
\mathcal{P}^{1}=\frac{1}{2}\left(\theta_{\mu \rho} \omega_{\nu \sigma}+\theta_{\mu \sigma} \omega_{\nu \rho}+\theta_{\nu \rho} \omega_{\mu \sigma}+\theta_{\nu \sigma} \omega_{\mu \rho}\right), \\
\mathcal{P}_{s}^{0}=\frac{1}{D-1} \theta_{\mu \nu} \theta_{\rho \sigma}, \\
\mathcal{P}_{w}^{0}=\omega_{\mu \nu} \omega_{\rho \sigma}, \\
\mathcal{P}_{s w}^{0}=\frac{1}{\sqrt{D-1}} \theta_{\mu \nu} \omega_{\rho \sigma}, \\
\mathcal{P}_{w s}^{0}=\frac{1}{\sqrt{D-1}} \omega_{\mu \nu} \theta_{\rho \sigma},
\end{gathered}
$$

where

$$
\theta_{\mu \nu}=\eta_{\mu \nu}-\frac{k_{\mu} k_{\nu}}{k^{2}}
$$

and

$$
\omega_{\mu \nu}=\frac{k_{\mu} k_{\nu}}{k^{2}} .
$$

we have

$$
a(\square) h_{\mu \nu} \rightarrow a\left(-k^{2}\right)\left[\mathcal{P}^{2}+\mathcal{P}^{1}+\mathcal{P}_{s}^{0}+\mathcal{P}_{w}^{0}\right] h,
$$

$$
b(\square) \partial_{\sigma} \partial_{(\nu} h_{\mu)}^{\sigma} \rightarrow-b\left(-k^{2}\right) k^{2}\left[\mathcal{P}^{1}+2 \mathcal{P}_{w}^{0}\right] h,
$$

$$
\begin{gathered}
c(\square)\left(\eta_{\mu \nu} \partial_{\rho} \partial_{\sigma} h^{\rho \sigma}+\partial_{\mu} \partial_{\nu} h\right) \\
\rightarrow-c\left(-k^{2}\right) k^{2}\left[2 \mathcal{P}_{w}^{0}+\sqrt{D-1}\left(\mathcal{P}_{s w}^{0}+\mathcal{P}_{w s}^{0}\right)\right] h, \\
\eta_{\mu \nu} d(\square) h \rightarrow d\left(-k^{2}\right)\left[(D-1) \mathcal{P}_{s}^{0}+\mathcal{P}_{w}^{0}\right. \\
\left.+\sqrt{D-1}\left(\mathcal{P}_{s w}^{0}+\mathcal{P}_{w s}^{0}\right)\right] h, \\
f(\square) \partial^{\sigma} \partial^{\rho} \partial_{\mu} \partial_{\nu} h_{\rho \sigma} \rightarrow f\left(-k^{2}\right) k^{4} \mathcal{P}_{w}^{0} h .
\end{gathered}
$$

Hence,

$$
\begin{gathered}
a k^{2} \mathcal{P}^{2} h=\kappa \mathcal{P}^{2} \tau \Rightarrow \mathcal{P}^{2} h=\kappa\left(\frac{\mathcal{P}^{2}}{a k^{2}}\right) \tau, \\
(a+b) k^{2} \mathcal{P}^{1} h=\kappa \mathcal{P}^{1} \tau \Rightarrow \mathcal{P}^{1} \tau=0, \\
(a+(D-1) d) k^{2} \mathcal{P}_{s}^{0} h+(c+d) k^{2} \sqrt{D-1} \mathcal{P}_{s w}^{0} h=\kappa \mathcal{P}_{s}^{0} \tau, \\
(c+d) k^{2} \sqrt{D-1} \mathcal{P}_{w s}^{0} h+(a+2 b+2 c+d+f) k^{2} \mathcal{P}_{w}^{0} h \\
=\kappa \mathcal{P}_{w}^{0} \tau .
\end{gathered}
$$

So,

$$
\begin{gathered}
(a+(D-1) d) k^{2} \mathcal{P}_{s}^{0} h=\kappa \mathcal{P}_{s}^{0} \tau \Rightarrow \mathcal{P}_{s}^{0} h \\
=\kappa \frac{\mathcal{P}_{s}^{0}}{(a+(D-1) d) k^{2}} \tau, \\
(a+2 b+2 c+d+f) k^{2} \mathcal{P}_{w}^{0} h \\
=\kappa \mathcal{P}_{w}^{0} \tau \Rightarrow \mathcal{P}_{w}^{0} h=\kappa \frac{\mathcal{P}_{w}^{0}}{(a+2 b+2 c+d+f) k^{2}} \tau,
\end{gathered}
$$

where we have used the constraints given by (A10)-(A12), and note that the denominator corresponding to the $P_{w}^{0}$ spin projector vanishes so that there is no $w$-multiplet. Thus, the $D$-dimensional propagator is given by

$$
\Pi\left(-k^{2}\right)=\frac{\mathcal{P}^{2}}{k^{2} a\left(-k^{2}\right)}+\frac{\mathcal{P}_{s}^{0}}{k^{2}\left(a\left(-k^{2}\right)-(D-1) c\left(-k^{2}\right)\right)} .
$$

Assuming $f(\square)=0 \Rightarrow a(\square)=c(\square)$, so as not to introduce any scalar propagating degrees of freedom, we find

$$
\Pi=\frac{1}{k^{2} a\left(-k^{2}\right)}\left(\mathcal{P}^{2}-\frac{1}{D-2} \mathcal{P}_{s}^{0}\right)
$$


[1] C. M. Will, The confrontation between general relativity and experiment, Living Rev. Relativity 17, 4 (2014).

[2] E. G. Adelberger, B. R. Heckel, S. A. Hoedl, C. D. Hoyle, D. J. Kapner, and A. Upadhye, Particle-Physics Implications of a Recent Test of the Gravitational Inverse-Square Law, Phys. Rev. Lett. 98, 131104 (2007).

[3] S. W. Hawking and G. F. R. Ellis, The Large Scale Structure of Space-Time, Cambridge Monographs on Mathematical Physics (Academic, New York, 1975), ISBN 9780521099066.

[4] J. D. Bekenstein, Black holes and entropy, Phys. Rev. D 7, 2333 (1973); Black holes and the second law, Lett. Nuovo Cimento 4, 737 (1972).

[5] S. W. Hawking, Black hole explosions?, Nature (London) 248, 30 (1974); Particle creation by black holes, Commun. Math. Phys. 43, 199 (1975).

[6] R. M. Wald, Black hole entropy is the Noether charge, Phys. Rev. D 48, R3427 (1993); V. Iyer and R. M. Wald, Some properties of the Noether charge and a proposal for dynamical black hole entropy, Phys. Rev. D 50, 846 (1994).

[7] T. Jacobson, G. Kang, and R. C. Myers, On black hole entropy, Phys. Rev. D 49, 6587 (1994).

[8] J. M. Maldacena, The Large-N Limit of superconformal field theories and supergravity, Int. J. Theor. Phys. 38, 1113 (1999).

[9] G. t Hooft, Dimensional reduction in quantum gravity, arXiv:gr-qc/9310026.

[10] L. Susskind, The world as a hologram, J. Math. Phys. (N.Y.) 36, 6377 (1995).

[11] K. S. Stelle, Renormalization of higher-derivative quantum gravity, Phys. Rev. D 16, 953 (1977).

[12] T. Biswas, E. Gerwick, T. Koivisto, and A. Mazumdar, Towards Singularity and Ghost-free Theories of Gravity, Phys. Rev. Lett. 108, 031101 (2012).

[13] T. Biswas, T. Koivisto, and A. Mazumdar, Nonlocal theories of gravity: the flat space propagator, arXiv:1302.0532.

[14] S. Talaganis, T. Biswas, and A. Mazumdar, Towards understanding the ultraviolet behavior of quantum loops in infinite-derivative theories of gravity, Classical Quantum Gravity 32, 215017 (2015).

[15] E. Tomboulis, Renormalizability and asymptotic freedom in quantum gravity, Phys. Lett. 97B, 77 (1980); E. T. Tomboulis, Superrenormalizable gauge and gravitational theories, arXiv:hep-th/9702146.

[16] L. Modesto, Super-renormalizable quantum gravity, Phys. Rev. D 86, 044005 (2012).

[17] A. Conroy, A. Mazumdar, and A. Teimouri, Wald Entropy for Ghost-Free, Infinite Derivative Theories of Gravity, Phys. Rev. Lett. 114, 201101 (2015).
[18] T. Biswas, A. Mazumdar, and W. Siegel, Bouncing universes in string-inspired gravity, J. Cosmol. Astropart. Phys. 03 (2006) 009.

[19] T. Biswas, A. Conroy, A. S. Koshelev, and A. Mazumdar, Generalized ghost-free quadratic curvature gravity, Classical Quantum Gravity 31, 015022 (2014); 31, 159501 (2014).

[20] H. J. Schmidt, Variational derivatives of arbitrarily high order and multi-inflation cosmological models, Classical Quantum Gravity 7, 1023 (1990).

[21] P. Van Nieuwenhuizen, On ghost-free tensor Lagrangians and linearized gravitation, Nucl. Phys. B60, 478 (1973).

[22] A. A. Tseytlin, On singularities of spherically symmetric backgrounds in string theory, Phys. Lett. B 363, 223 (1995).

[23] W. Siegel, Stringy gravity at short distances, arXiv:hep-th/ 0309093.

[24] V. P. Frolov, A. Zelnikov, and T. de Paula Netto, Spherical collapse of small masses in the ghost-free gravity, J. High Energy Phys. 06 (2015) 107.

[25] V. P. Frolov, Mass-Gap for Black Hole Formation in Higher Derivative and Ghost-free Gravity, Phys. Rev. Lett. 115, 051102 (2015).

[26] F. R. Tangherlini, Schwarzschild field in $\mathrm{n}$ dimensions and the dimensionality of space problem, Nuovo Cimento 27, 636 (1963).

[27] R. Emparan and H. S. Reall, Black holes in higher dimensions, Living Rev. Relativity 11, 6 (2008).

[28] T. Biswas, A. S. Koshelev, A. Mazumdar, and S. Y. Vernov, Stable bounce and inflation in non-local higher derivative cosmology, J. Cosmol. Astropart. Phys. 08 (2012) 024.

[29] T. Biswas, T. Koivisto, and A. Mazumdar, Towards a resolution of the cosmological singularity in non-local higher derivative theories of gravity, J. Cosmol. Astropart. Phys. 11 (2010) 008.

[30] B. Craps, T. De Jonckheere, and A. S. Koshelev, Cosmological perturbations in non-local higher-derivative gravity, J. Cosmol. Astropart. Phys. 11 (2014) 022; A. S. Koshelev and S. Y. Vernov, Cosmological solutions in nonlocal models, Phys. Part. Nucl. Lett. 11, 960 (2014).

[31] D. Chialva and A. Mazumdar, Cosmological implications of quantum corrections and higher-derivative extension, Mod. Phys. Lett. A 30, 1540008 (2015).

[32] A. Conroy, A. S. Koshelev, and A. Mazumdar, Geodesic completeness and homogeneity condition for cosmic inflation, Phys. Rev. D 90, 123525 (2014).

[33] F. Rief, Fundamentals of Statistical and Thermal Physics (McGraw-Hill Science, New York, 1965). 